\title{
Um Novo Modelo Multidimensional da Perspetiva Temporal
}

\author{
A New Multidimensional Model of Time Perspective
}

\author{
Victor E.C. Ortuño ${ }^{1}$, Isabel Nunes Janeiro ${ }^{2}$, Maria Paula Paixão ${ }^{3}$, Cristina Esteves ${ }^{4}$ e Pedro \\ Cordeiro $^{5}$
}

\section{Resumo}

A Perspetiva Temporal é uma dimensão cognitivo-motivacional. Um dos modelos com maior difusão na atualidade é apresentado por Zimbardo e Boyd (1999). Contudo, é importante referir que este modelo não considera a influência da temporalidade futura negativa, apesar de esta ser referida como fundamental por Lewin (1965). São três os objetivos deste estudo: determinar a estrutura fatorial i) do Zimbardo Time Perspective Inventory (ZTPI), ii) da Transcendental-Future Time Perspective Scale (TFTPS) através de Modelação de Equações Estruturais e iii) apresentar um novo modelo multidimensional no qual seja considerada a dimensão negativa da temporalidade futura. Os resultados com ambos os instrumentos foram coerentes com a teoria estabelecida, assim como também permitem sugerir um novo modelo formado por sete dimensões temporais.

Palavras-chave: perspetiva temporal, ZTPI, TFTPS, futuro negativo, temporalidade subjetiva, modelação de equações estruturais

\begin{abstract}
Time Perspective is a cognitive-motivational dimension. Currently, Zimbardo \& Boyd's (1999) model is one of the most cited, still, it doesn't consider the influence of future negative temporality, which is referred by Lewin (1965) as fundamental for human understanding. This study has three objectives, to determine i) Zimbardo Time Perspective Inventory (ZTPI) and ii) Transcendental-Future Time Perspective Scale (TFTPS) factor structures through Structural Equation Modeling and iii) to establish a new multidimensional model which includes the negative future dimension. Results with both instruments showed a structure coherently aligned with current theory, suggesting a new model formed by seven temporal dimensions.
\end{abstract}

Keywords: time perspective, ZTPI, TFTPS, negative future, subjective temporality, structural equation modeling

\footnotetext{
${ }^{1}$ Universidad de la República (Professor Adjunto) e Universidade de Coimbra (Investigador). Faculdade de Psicología. Calle Dr Tristán Narvaja 1674, Montevideo, Uruguai. Tel.: +351917334130. E-mail: victortuno@gmail.com / victortuno.netii.net / @ victortuno

${ }^{2}$ Universidade de Lisboa (Professora Auxiliar). Faculdade de Psicologia, Alameda da Universidade, 1649-013 Lisboa, Portugal. Tel.: +351966110768. E-mail: injaneiro@psicologia.ulisboa.pt

3 Universidade de Coimbra (Professora Associada). Rua do Colégio Novo, 3000-115 Coimbra, Portugal. Tel.: +351919371460. E-mail: mppaixao@fpce.uc.pt

${ }^{4}$ Universidade de Coimbra (Investigadora). Rua do Colégio Novo, 3000-115 Coimbra, Portugal. Tel.: +351933641684 . E-mail: cristina.m.esteves@gmail.com

${ }^{5}$ Universidade de Coimbra (Investigador). Rua do Colégio Novo, 3000-115 Coimbra, Portugal. Tel.: +351966664542 .

E-mail: pedrcordeiro@gmail.com
} 


\section{Introdução}

Waste not your time, so fast it flies; Method will teach you time to win; Hence, my young friend, I would advise, With college logic to begin.

- Goethe

Os conceitos referentes às dimensões subjetivas do tempo têm tido um papel de destaque ao longo da história da Psicologia. Não obstante, alguns anos após os trabalhos de Nuttin e Lens (1985) verificou-se um refreamento na produção científica em torno da temporalidade subjetiva. Na última década um dos conceitos emergentes foi o de Perspetiva Temporal (PT). O recrudescer do interesse na PT tem sido fortemente atribuído à investigação de Zimbardo e Boyd (1999), que não só fazem uma atualização da teoria temporal, como também apresentam um novo método de avaliação da PT. Este novo paradigma da investigação da temporalidade originou inúmeros estudos empíricos, com mais de 1600 citações registadas na base de dados Google Scholar.

O modelo de PT de Zimbardo e Boyd (1999) assenta fundamentalmente no quadro teórico proposto por Lewin (1965). Lewin argumenta que tanto a cognição como o comportamento humano são influenciados pelas dimensões subjetivas da temporalidade passada e futura, já que ambas se encontram ativas no momento presente. De acordo com Zimbardo e Boyd (1999) a PT é um processo não consciente, no qual os objetos e acontecimentos, tanto pessoais como sociais, são categorizados, organizados e acedidos tendo em conta cinco categorias temporais: O Passado Positivo está relacionado com uma visão positiva e calorosa em relação aos acontecimentos passados. O Passado Negativo refere-se a acontecimentos passados vividos de forma negativa e traumática. O Presente Hedonista caracteriza-se por uma forte procura de sensações e experiências novas, sem grande preocupação pelas suas possíveis consequências. O Presente Fatalista representa uma atitude de desespero relativamente ao presente, em que os acontecimentos são percebidos como sendo devidos a causas externas, que estão fora do controlo do próprio indivíduo (e.g., o governo, deus, entre outros). Por fim, a dimensão do Futuro reflete uma postura de antecipação e planificação do futuro e onde as gratificações do momento presente são geralmente colocadas num segundo plano. Mais recentemente foi adicionada também a dimensão de Futuro Transcendental, que avalia as crenças que os indivíduos possuem acerca do futuro após a morte do corpo físico e da forma como esta vivência post-mortem pode ser influenciada pelos nossos atos em vida (Boyd \& Zimbardo, 1997). Estas categorias ou marcos temporais funcionam como estruturas que permitem dar sentido, ordem e coerência a toda a experiência existencial dos indivíduos. $\mathrm{O}$ mesmo será dizer que representam um quadro organizativo e interpretativo de referência para os objetos motivacionais dos indivíduos, sejam eles internos ou externos (Nuttin \& Lens, 1985).

O modelo de Zimbardo e Boyd (1999) tem estimulado o estudo da PT em vários contextos são diversos os estudos desenvolvidos no espaço Iberoamericano. Corral-Verdugo, Fraijo-Sing e Pinheiro (2006) demostraram como comportamentos de conservação de água se encontram relacionados negativamente com a Perspetiva Temporal de Presente e positivamente com a Perspectiva Temporal de Futuro (PTF). Na mesma linha, Milfont e Gouveia (2006) suportam a associação positiva entre a PTF e atitudes de conservação ambiental, bem como a associação negativa entre a presença de valores altruístas e o Passado Negativo. Já Ferrari e Diaz-Morales (2007) referem como o Presente Fatalista está positivamente associado à Procrastinação de tipo evitamento, enquanto o Presente Hedonista aparece associado positivamente com a Procrastinação de tipo estimulante. Por último, no contexto escolar, Carvalho (2015) indica que a PTF é um preditor positivo da adaptação escolar em adolescentes.

Porém, alguns autores referem que uma franja considerável dos estudos realizados em torno da PT apresenta uma lacuna fundamental, que se prende com o facto de não considerar a influência da temporalidade futura negativa (Holman \& Silver, 2005; Ortuño \& Vásquez, 2013; Zaleski, 1996). Por sua vez, Trommsdorff (1983) afirma que a componente afetiva da PTF possui maior influência nas cognições e comportamentos do que a componente cognitiva da PTF. Numa 
tentativa de ultrapassar estas limitações Carelli, Wiberg e Wiberg (2011) defendem que a avaliação da PT não se deve cingir apenas aos aspetos positivos da temporalidade futura, mas também deve incluir os aspetos negativos como o medo, a preocupação, a ansiedade e a incerteza.

A este propósito, é importante considerar que, na formulação original do conceito de PT, Lewin $(1939,1965)$ afirmou que os indivíduos são influenciados pela forma como percebem e constroem o futuro, isto é, as suas expectativas e esperanças, mas também, e em igual medida, pelos seus medos. Para Zaleski (1996) a PTF constitui um espaço aberto para diferentes processos cognitivos e emocionais, de cariz positivo ou negativo, que não são mutuamente exclusivos.

Desta forma, e em conformidade com os autores supramencionados, consideramos que uma abordagem compreensiva da temporalidade futura deve integrar uma dimensão de futuro negativo. Esta posição é sustentada, ao nível empírico, pela diversidade de estudos que tem demonstrado, de forma consistente, a relevância do futuro negativo em diversos processos cognitivos e comportamentais. Por exemplo, Nobre e Janeiro (2010) apresentam correlações negativas e significativas entre o futuro negativo, o Bem-estar Escolar e a Atitude Académica. Em relação aos estilos de tomada de decisão, Carelli e colaboradores (2011) verificam uma forte associação positiva entre o Futuro Negativo e os estilos decisionais dependente e evitante. Mais recentemente, Ortuño e colaboradores (2013d) apresentaram o futuro negativo como a única dimensão temporal com poder preditivo do Equilíbrio Emocional, os quais se associam negativamente. Ortuño e Vásquez (2013) utilizando modelação por equações estruturais, demonstraram a influência negativa do Futuro Negativo na Auto-estima traço.

\section{Objetivos do Estudo}

O presente trabalho dá continuidade ao esforço iniciado por Ortuño, Gomes, Paixão e Janeiro (2013c) e Ortuño (2014), no sentido de validar um modelo mais compreensivo de temporalidade subjetiva que inclua a dimensão de temporalidade futura negativa. $\mathrm{O}$ presente trabalho desenvolve-se em três estudos, relacionados com os seus três objetivos centrais deste trabalho: i) testar a estrutura fatorial da versão portuguesa do Zimbardo Time Perspective Inventory (ZTPI). ii) testar a estrutura fatorial da versão portuguesa da Transcendental-Future Time Perspective scale (TFTPS), e iii) testar um modelo multidimensional da Perspetiva Temporal (PT) que inclui uma dimensão de futuro negativo. A estrutura destes instrumentos, assim como do modelo final, serão testadas com técnicas estatísticas mais robustas do que as que foram utilizadas nos estudos prévios, mais especificamente a modelação de equações estruturais.

\section{Estudo 1}

\section{Método}

\section{Participantes}

Foi utilizada uma amostra composta por 816 estudantes do Mestrado Integrado em Psicologia da Universidade de Coimbra $(n=629,77.1 \%)$, da Universidade de Lisboa $(n=107,13.1 \%)$ e da Universidade do Porto $(n=80,9.8 \%)$. Destes, 708 (87.2\%) são do género feminino e 104 (12.8\%) do género masculino, tendo idades compreendidas entre os 17 e os 61 anos $(M=20.10, D P=4.97)$.

\section{Instrumentos}

Foi utilizado o Zimbardo Time Perspective Inventory (ZTPI, Zimbardo \& Boyd, 1999), o qual é composto por 56 afirmações cotadas em um formato de resposta Likert de 5 pontos, no qual 1 $=$ Nada, $3=$ Nem muito nem pouco e $5=$ Totalmente. O ZPTI apresenta uma estrutura multidimensional composta por 5 dimensões temporais: a) Passado Positivo, "Eu fico nostálgico acerca da minha infância" ( $S^{2}=6.02 \%$, $\alpha=.68)$. b) Passado Negativo, "Continuo a reviver no meu pensamento as experiências dolorosas do passado" $\quad\left(S^{2}=7.85 \%, \quad \alpha=.80\right) . \quad$ c) Presente Hedonista, "Eu corro riscos para sentir emoção na minha vida" $\left(S^{2}=8.37 \%, \alpha=.79\right)$. d) Presente Fatalista, "O meu percurso de vida é controlado por forças sobre as quais eu não tenho influência" $\left(S^{2}=6.42 \%, \alpha=.66\right)$. E e) Futuro, "Cumprir prazos para amanhã e fazer qualquer outro trabalho necessário está primeiro do que a diversão de 
hoje à noite" ( $\left.S^{2}=6.57 \%, \alpha=.74\right)$. Estes resultados pertencem à versão portuguesa do ZTPI (Ortuño \& Gamboa, 2008, 2009) a qual replica tanto a estrutura fatorial como as características psicométricas do ZTPI original (Zimbardo \& Boyd, 1999), e que é também equivalente a outras versões internacionais (Sircova et al., 2014).

\section{Procedimentos}

Todos os dados foram recolhidos nas Faculdades de Psicologia e de Ciências da Educação da Universidade de Coimbra e da Universidade do Porto e na Faculdade de Psicologia da Universidade de Lisboa. Dos 56 itens, 48 apresentaram valores omissos, embora nunca mais de sete observações por item $(0.9 \%$ das respostas totais por item). Para lidar com os valores omissos decidiu-se calcular o teste Little's Missing Completely At Random (MCAR). Subsequentemente, para substituir os valores omissos foi utilizado o algoritmo ExpectationMaximization (EM) nas dimensões Passado Negativo, Presente Fatalista e Futuro, já que foram aquelas a apresentar um valor $p>.05$ no teste MCAR (ver Quadro 1). Em relação às outras duas dimensões (Passado Positivo e Presente Hedonista), os participantes que ainda tinham algum valor omisso no ZTPI foram eliminados da base de dados. Após estes refinamentos, a amostra final compreendeu um total de 795 participantes.

Quadro 1. Resultados do teste de Little Missing Completely at Random (MCAR) para cada subescala do ZTPI

\begin{tabular}{lccc}
\hline Sub-escala & $x^{2}$ & $d f$ & $p$ \\
\hline Passado Positivo & 87.72 & 47 & .00 \\
Passado Negativo & 96.14 & 93 & .39 \\
Presente Hedonista & 191.72 & 160 & .04 \\
Presente Fatalista & 67.61 & 57 & .16 \\
Futuro & 75.22 & 79 & .60 \\
\hline
\end{tabular}

\section{Resultados}

\section{Normalidade da distribuição}

Previamente à realização da AFC, foi testada a normalidade da distribuição. Os valores da assimetria e da curtose não indicam qualquer violação do princípio da normalidade univariada (sk<3, ku<10, Marôco, 2010).

\section{Análise fatorial confirmatória}

Foram testados três modelos estruturais, considerando os valores de corte propostos por Marôco (2010) para os indices de ajustamento utilizados: $X^{2} d f<5$, CFI e GFI > .90, PCFI e PGFI > .60 e RMSEA < .05. O Modelo 1 foi composto pelos 56 itens do ZTPI, organizados nas suas respetivas cinco dimensões temporais sem adicionar qualquer outro parâmetro. No Modelo 2 foram introduzidas trajetórias correlacionais entre os erros do itens com valores $\mathrm{IM}>11$.

O Modelo 1 não apresentou bons resultados na maioria dos índices de ajustamento geral (ver Quadro 2).

O passo seguinte consistiu em eliminar os itens com resultados baixos ao nível da sua carga fatorial $(\lambda<.50)$ e da sua confiabilidade $\left(R^{2}=.25\right)$. Este procedimento foi levado a cabo de forma faseada: a cada item que era retirado, eram novamente calculados e analisados os índices de ajustamento global e indicadores dos itens. O diagrama deste modelo (Modelo 3) pode ser consultado na Figura 1.

Através da análise comparativa dos três modelos testados e cujos índices de ajustamento se encontram detalhadamente descritos no Quadro 2 , é possível verificar que o Modelo 3 , referente a uma versão reduzida de 25 itens do ZTPI, é o modelo mais estável e parcimonioso (Marôco, 2010).

Quadro 2. Índices de ajustamento global dos modelos testados (ZTPI)

\begin{tabular}{cccccccccccc}
\hline & $X^{2}$ & $d f$ & $\Delta X^{2}$ & $\Delta d f$ & $X^{2} / d f$ & AIC & CFI & PCFI & GFI & PGFI & RMSEA \\
\hline Modelo 1 & 5675 & 1474 & - & - & 3.85 & 5919 & .62 & .59 & .77 & .71 & .06 \\
Modelo 2 & 4925.28 & 1462 & 749.72 & 12 & 3.37 & 5193.28 & .69 & .65 & .80 & .73 & .06 \\
Modelo 3 & 6666.20 & 254 & 5008.8 & 1220 & 2.62 & 808.20 & .92 & .78 & .94 & .73 & .05
\end{tabular}

Nota. $\Delta X^{2}$ e $\Delta d f$ foram obtidos através de comparação com o Modelo 1. 


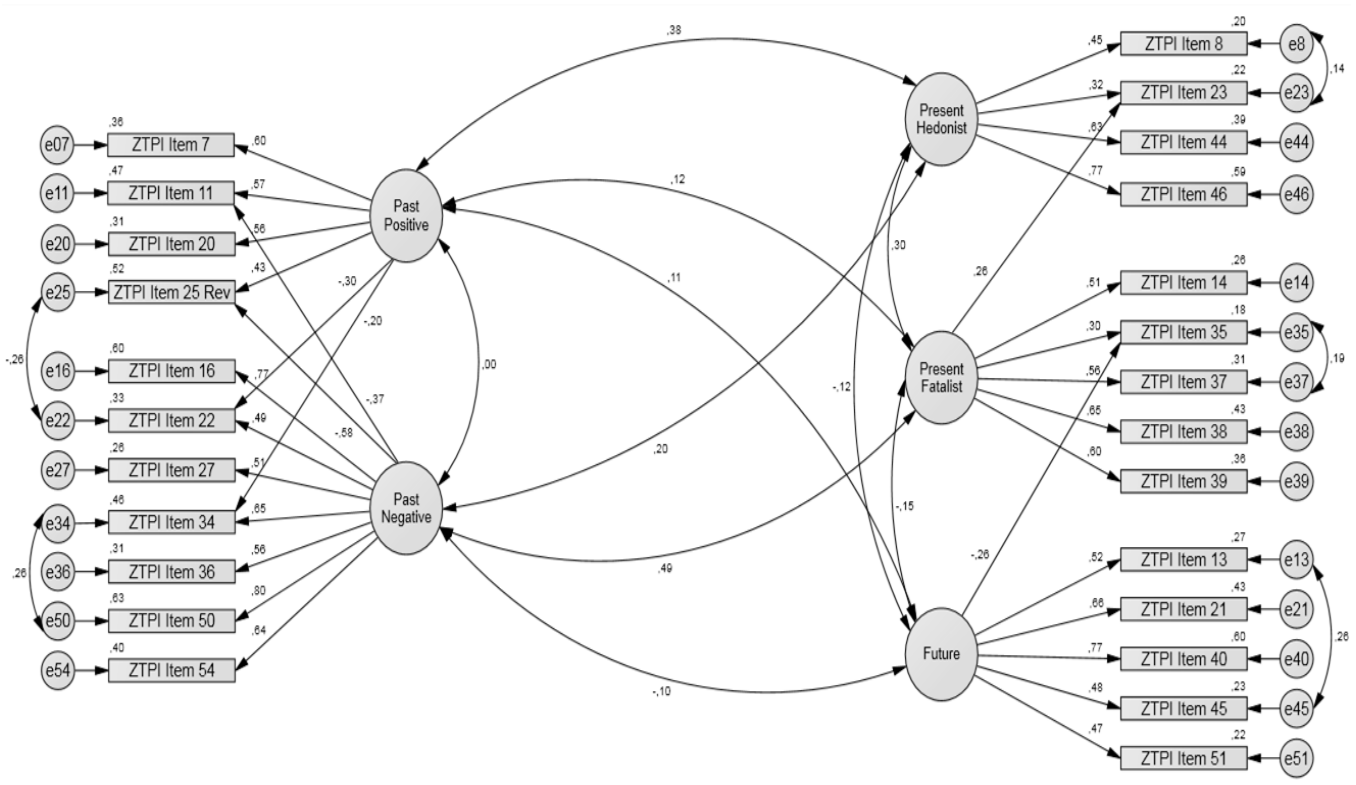

Figura 1. Path Diagram da versão breve do ZTPI (Modelo 3)

\section{Discussão}

O ZTPI tem sido um instrumento altamente enaltecido pela comunidade científica na última década. A sua estrutura fatorial tem sido replicada em diversos idiomas e países (Anagnostopoulos \& Griva, 2012; Apostolidis \& Fieulaine, 2004; DiazMorales, 2006; Leite \& Pasquali, 2008; Liniauskaite \& Kairys, 2009; Košt’ál, KlicperováBaker, Lukavská, \& Lukavský, 2015; Orkibi, 2015; Ortuño \& Gamboa, 2009). Ainda assim, alguns autores manifestam cautela quando a análise estrutural é feita através de uma metodologia confirmatória. Os resultados neste âmbito têm variado entre valores aceitáveis (Anagnostopoulos \& Griva, 2012; Apostolidis \& Fieulaine, 2004; Cretu, 2012), intermédios (Carelli et al., 2011) ou não satisfatórios (Liniauskaite \& Kairys, 2009; Milfont, Andrade, Belo, \& Pessoa, 2008). Contudo, não é possível fazer-se uma comparação direta entre estes resultados, dado que vários destes estudos apresentam abordagens "personalizadas" ou "restritas" do ZTPI. Alguns optaram por avaliar exclusivamente a estrutura original (Anagnostopoulos \& Griva, 2012), enquanto outros decidiram remover itens (Apostolidis \& Fieulaine, 2004) ou, inclusive, incluir novos (Carelli et al., 2011). O objetivo deste estudo foi analisar a estrutura fatorial da versão portuguesa do ZTPI através de AFC. O modelo composto pelos 56 itens (Modelo 1) não atingiu valores adequados de ajustamento global. A versão modificada (Modelo 2), na qual os resíduos de diversas co-variâncias foram correlacionados (utilizando como critério o índice de modificação), também não atingiu os níveis recomendados para um bom ajustamento. Finalmente foi testado um modelo formado por 25 itens (Modelo 3), o qual apresentou índices de ajustamentos adequados. Apenas o teste do chi quadrado apresentou um resultado inadequado, já que o valor $p$ é estatisticamente significativo. Ainda assim, é importante considerar que este teste é altamente sensível a amostras de grande número (Hooper, Coughlan, \& Mullen, 2008), o que pode estar na base deste resultado. Como é possível verificar na Figura 1, todos os itens apresentaram uma boa carga fatorial e elevados níveis de fiabilidade.

Devido ao número de itens retirado nesta versão do ZTPI é possível considerá-la uma versão reduzida do mesmo. Em relação a este assunto Gupta, Hershey e Gaur (2012) mencionaram que diversas tentativas já foram realizadas até a data, mas que os resultados são divergentes, já que cada estudo valida um conjunto diferente de itens. Azevedo, Teixeira e Paúl (2012) alertam para a necessidade de uma versão reduzida para utilização em grupos específicos (tais como idosos). Contudo, uma primeira tentativa para desenvolver uma versão 
breve do ZTPI em contexto Português (Imaginário, Oyanadel, Gamboa, \& Jesus, 2011) mostrou-se insatisfatória devido a uma baixa fiabilidade.

Pelo contrario, a presente proposta para uma versão reduzida do ZTPI apresenta bons indicadores psicométricos, assim como uma estrutura fatorial muito similar à do ZTPI original, ainda que sejam removidos 31 dos seus 56 itens. Para finalizar, consideramos uma melhoria no estudo confirmatório do ZTPI que sejam consideradas as cargas fatoriais cruzadas que alguns itens apresentam. São caso disto os itens 22 "No passado, tive a minha dose de maus-tratos e rejeição" e 34 "É difícil para mim esquecer imagens desagradáveis da minha juventude", os quais estão associados negativamente com o fator Passado Positivo. Os itens 11 "Fazendo um balanço, há mais memórias boas do que más para recordar no meu passado" e 25 "O passado trazme demasiadas más memórias, nas quais eu prefiro não pensar", os quais apresentam uma relação negativa com o fator Passado Negativo. O item 23 "Tomo as minhas decisões de acordo com a inspiração do momento", o qual se encontra positivamente associado com o fator Presente Fatalista. E por último, o item 35 "Se tenho que pensar nos objetivos, resultados e produtos das minhas atividades, isso tira-me o prazer e estraga o decorrer do processo" o qual apresenta uma associação negativa com o fator Futuro. Tendo em conta o conteúdo destes itens, parece-nos lógico considerar estas novas cargas fatoriais.

\section{Estudo 2}

\section{Método}

\section{Participantes}

Um total de 346 participantes fizeram parte deste estudo. As idades estão compreendidas entre os 17 e os $54 \operatorname{anos}(M=19.87, D P=4.27)$. Em relação ao género, $313(90.7 \%)$ participantes pertencem ao género feminino enquanto, 32 $(9.3 \%)$ ao género masculino. Todos os participantes são estudantes universitários do Mestrado Integrado em Psicologia, distribuídos pelos seguintes anos: 186 (54.2\%) do primeiro ano, 77 (22.4\%) do segundo ano, 77 (22.4\%) do terceiro ano, 1 (.3\%) do quarto ano e $2(.6 \%)$ do quinto ano.

\section{Instrumentos}

Foi utilizada a Transcendental-Future Time Perspective Scale (TFTPS, Boyd \& Zimbardo, 1997) para avaliar as atitudes e crenças em relação ao futuro imaginado que acontece após a morte do corpo físico, isto é, a Perspetiva Temporal de Futuro Transcendental. É uma escala unidimensional, composta por 10 itens (com uma escala de resposta em formato Likert de 5 pontos, onde $1=$ Nada, $3=$ Nem muito nem pouco e $5=$ Totalmente) tais como "Serei responsabilizado pelas minhas acções na terra quando morrer”. A estrutura fatorial da TFTPS foi testada originalmente em conjunto com os 56 itens do ZTPI. Nesta análise, o fator composto pelos itens da TFTPS explicou $10 \%$ da variância total do modelo, sendo o fator com mais variância explicada e com uma boa consistência interna $(\alpha=.87)$. Na sua versão portuguesa (Ortuño, Paixão, \& Janeiro, 2013a), a TFTPS apresentou também uma boa consistência interna $(\alpha=.87)$, utilizando 9 dos 10 itens originais da TFTPS.

\section{Procedimentos}

Todos os dados foram recolhidos na Faculdade de Psicologia e de Ciências da Educação da Universidade de Coimbra, Portugal. Todos os participantes que apresentaram valores omissos em algum dos itens da TFTPS foram retirados da base de dados, já que não foi possível utilizar a técnica EM devido ao resultado no teste MCAR ter tido um valor $p<.05$.

\section{Resultados}

Primeiramente foi verificado o requisito da normalidade da distribuição. Tanto o valor de assimetria como o da curtose indicam uma distribuição normal $(s k<3$ e $k u<10)$. Para verificar a estrutura fatorial da TFTPS foi realizada uma AFC com Estimativa por Máxima Verosimilhança, a qualidade do ajustamento de cada um dos modelos testados foi avaliada utilizando os mesmos índices de ajustamento apresentados no Estudo 1, assim como os pontos de corte propostos por Marôco (2010).

O primeiro modelo a ser testado (Modelo 1) é formado pelos 10 itens originais propostos por 
Quadro 3. Índices de ajustamento global dos modelos testados (TFTPS)

\begin{tabular}{cccccccccccc}
\hline & $X^{2}$ & $d f$ & $\Delta X^{2}$ & $\Delta d f$ & $X^{2} d f$ & AIC & CFI & PCFI & GFI & PGFI RMSEA \\
\hline Modelo 1 & 330.37 & 35 & - & - & 9.44 & 370.37 & .85 & .66 & .87 & .55 & .13 \\
Modelo 2 & 312.98 & 27 & 17.39 & 8 & 11.59 & 348.98 & .86 & .64 & .86 & .52 & .14 \\
Modelo 3 & 33.93 & 10 & 296.44 & 25 & 3.39 & 69.93 & .99 & .48 & .99 & .35 & .03 \\
\hline
\end{tabular}

Nota. $\Delta X^{2}$ e $\Delta d f$ foram obtidos através de comparação com o Modelo 1.

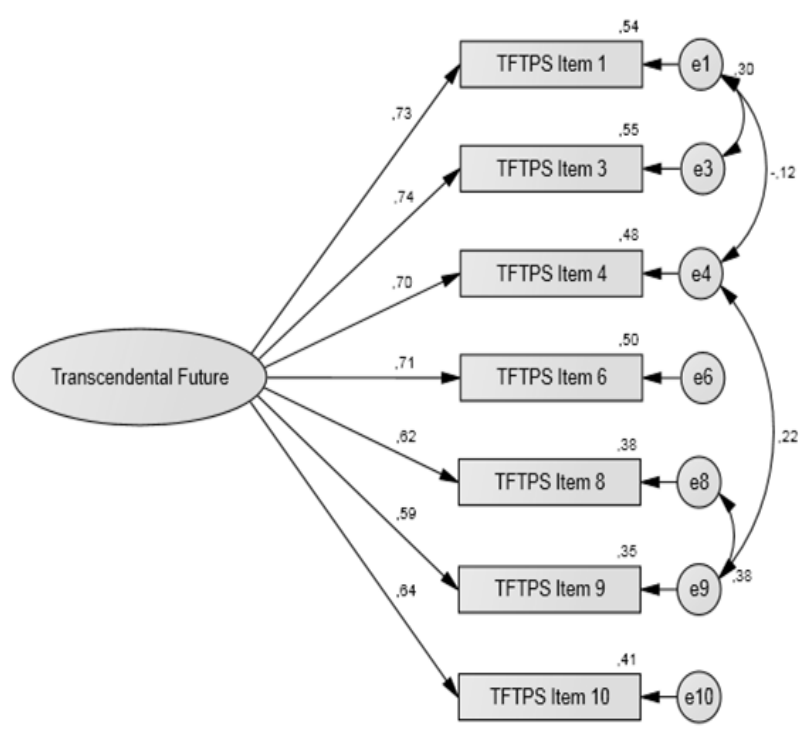

Figura 2. Path Diagram da TFTPS (Modelo 3)

Boyd e Zimbardo (1997) e organizados num único fator latente. Com este modelo não foi possível obter índices de ajustamento global aceitáveis (ver Quadro 3). Em relação aos itens, o item 5 apresentou resultados particularmente desfavoráveis na carga fatorial $(\lambda=.07, p=.134) \mathrm{e}$ na confiabilidade $\left(R^{2}=.01\right)$. Considerando este resultado, assim como as recomendações de Ortuño et al. (2013a) foi removido o Item 5. O Modelo 2 apresenta ligeiras melhorias em relação ao anterior (ver Quadro 3), ainda assim, consideramos que as estas não são expressivas e podem dever-se principalmente à própria simplificação do modelo e não a reais melhorias do seu ajustamento. Todos os itens apresentaram uma boa carga fatorial $(\lambda=.50)$ e confiabilidade $\left(R^{2}=.25\right)$ com a exceção do Item $7\left(\lambda=.43, R^{2}=.19\right)$.

Assim, foram efetuadas algumas modificações ao modelo. Dois itens foram removidos: o item 7 devido ao baixo valor na sua carga factorial e na sua confiabilidade. Já o item 2 foi eliminado devido ao seu elevado valor no Índice de
Modificação, já que apresentava uma elevada covariância com mais de metade dos itens. Para além disso, quatro trajetórias correlacionais foram adicionadas ao modelo, devido ao seu valor no Índice de Modificação (MI>11). Este modelo (Modelo 3) pode ser consultado na Figura 2 e no Quadro 3 é possível verificar em detalhe o ajustamento deste modelo, assim como a comparação com os dois modelos prévios.

O Modelo 3 apresentou um bom ajustamento aos dados (CFI=.99, GFI=.99, NFI=.99), assim como também todas as trajetórias apresentaram uma elevada significância estatística $(p<.001)$. Os itens também apresentaram cargas fatoriais elevadas $(\lambda \geq .50)$ e uma boa confiabilidade $\left(R^{2} \geq .25\right)$.

Deste modo, considerando os resultados presentes no Quadro 3, é possível verificar que o modelo composto por sete itens (Modelo 3) é o mais parcimonioso e o mais estável dos três modelos testados neste estudo. O Modelo 3 foi aquele que apresentou um valor mais baixo no Akaike's Information Criterion (AIC). Também as diferenças no valor de $X^{2}$, permitem indicar o Modelo 3 como aquele com melhor ajustamento (Modelo $3 \Delta X^{2}=296.44>$ Modelo $2 \Delta X^{2}=17.39$ ).

\section{Discussão}

Após testar três diferentes modelos em relação à estrutura da TFTPS, foi encontrado um modelo que pode ser considerado como adequado, o Modelo 3, que é composto por sete itens. Contudo, consideramos pertinente uma breve reflexão em relação ao conteúdo de dois dos itens que foram retirados do modelo, já que estes apresentam claramente uma posição relativa contra a ciência e as suas conclusões. Ortuño et al. (2013a) consideram que na cultura portuguesa não há espaço para posicionamentos de confronto 
entre a ciência e a religião. Assim, em conformidade com este trabalho, consideramos que os itens 5 e 7 apresentam pouca relação com o conteúdo da Perspetiva Temporal de Futuro Transcendental.

O modelo final apresentou uma elevada confiabilidade assim como elevadas cargas fatoriais. Todas as trajetórias definidas são estatisticamente significativas. Por último, considerando as recomendações de Marôco (2010), o ajustamento global do modelo aos dados pode ser considerado como elevado.

\section{Estudo 3}

\section{Método}

\section{Participantes}

Participaram neste estudo 215 participantes, dos quais 178 (82.8\%) são alunos do Mestrado Integrado em Psicologia. Os restantes são alunos dos cursos de Ciências da Educação ( $n=27$, $12.6 \%)$, Gestão $(n=1, \quad .5 \%), \quad$ Engenharia Electrónica $(n=1, .5 \%)$ e $8(3.7 \%)$ participantes que não eram alunos universitários. As instituições de ensino são a Universidade de Lisboa $(n=104,50.2 \%)$ e a Universidade de Coimbra $(n=103,49.8 \%)$. Todos os alunos eram estudantes no primeiro ano do curso. Em relação ao género, $164(76.3 \%)$ pertencem ao género feminino e 51 (23.7\%) ao género masculino. As idades dos participantes estão compreendidas entre os 17 e os 61 anos de idade $(M=19.97$, $D P=5.39)$.

\section{Instrumentos}

Além dos instrumentos previamente descritos, foram também utilizados neste estudo os itens relativos à dimensão de Futuro Negativo do Inventário de Perspetiva Temporal (IPT).

Foi utilizado o Inventário de Perspetiva Temporal (IPT, Janeiro, 2012), o mesmo é formado por 32 itens (com uma escala de resposta em formato de Likert de 7 pontos, onde 1 = Não corresponde e $7=$ Corresponde muito), o IPT é formado por quatro fatores, os quais representam quatro orientações temporais: Orientação Temporal de Passado $\left(S^{2}=7 \%, \alpha=.51\right)$; Orientação Temporal de Presente $\left(S^{2}=13 \%, \alpha=.76\right)$;
Orientação Temporal de Futuro $\left(S^{2}=16 \%, \alpha=.86\right)$ e Visão Ansiosa do Futuro ou Futuro Negativo $\left(S^{2}=8 \%, \alpha=.70\right)$. Neste estudo foram utilizados apenas os itens referentes à dimensão de Futuro Negativo (ex.: "Caminho para o futuro um pouco à deriva, não por opção mas porque não consigo parar"), os quais apresentaram uma consistência interna de $\alpha=.84$.

\section{Procedimentos}

O tratamento para os valores omissos consistiu primeiro no cálculo do teste Little's MCAR e posterior utilização do algoritmo EM para substituir os valores em falta. Este procedimento foi efetuado em cinco das sete dimensões em estudo. As duas dimensões nas quais não foi utilizado o algoritmo EM foram o Passado Positivo e o Futuro Transcendental, já que apresentaram um valor significativo $(p<.05)$ no teste MCAR. Desta forma, foram eliminados da base de dados quatro participantes, os quais apresentavam valores omissos em alguma dessas duas dimensões.

O modelo fatorial proposto de sete fatores, assim como as relações entre estas, foram testadas com recurso a Path Analysis e AFC. Após isto, foi efetuada uma Análise Fatorial Exploratória (AFE) de forma a determinar a quantidade de variância total explicada pelo modelo.

\section{Resultados}

O modelo que se apresenta de seguida pode ser considerado a evolução do trabalho apresentado previamente por Ortuño e colaboradores (2013c). As diferenças consistem i) na eliminação de alguns dos itens do ZTPI e da TFTPS devido aos seus indicadores psicométricos ao nível individual, mas também ao seu contributo para o ajustamento global dos modelos. ii) A correlação entre os resíduos de dois itens da TFTPS foram removidos devido à falta de significância estatística $\left(r_{\text {etf01-etf03 }}=.16, p=.151\right)$. iii) A trajetória regressional $\beta_{\text {PastPositive-Item34 }}=-.07$, $p=.35$, que fora proposta na AFC do ZTPI, foi removida devido à sua falta de significância estatística, assim como à falta de intensidade na associação. iv) Oito trajetórias correlacionais foram removidas devido a resultados não significativos 


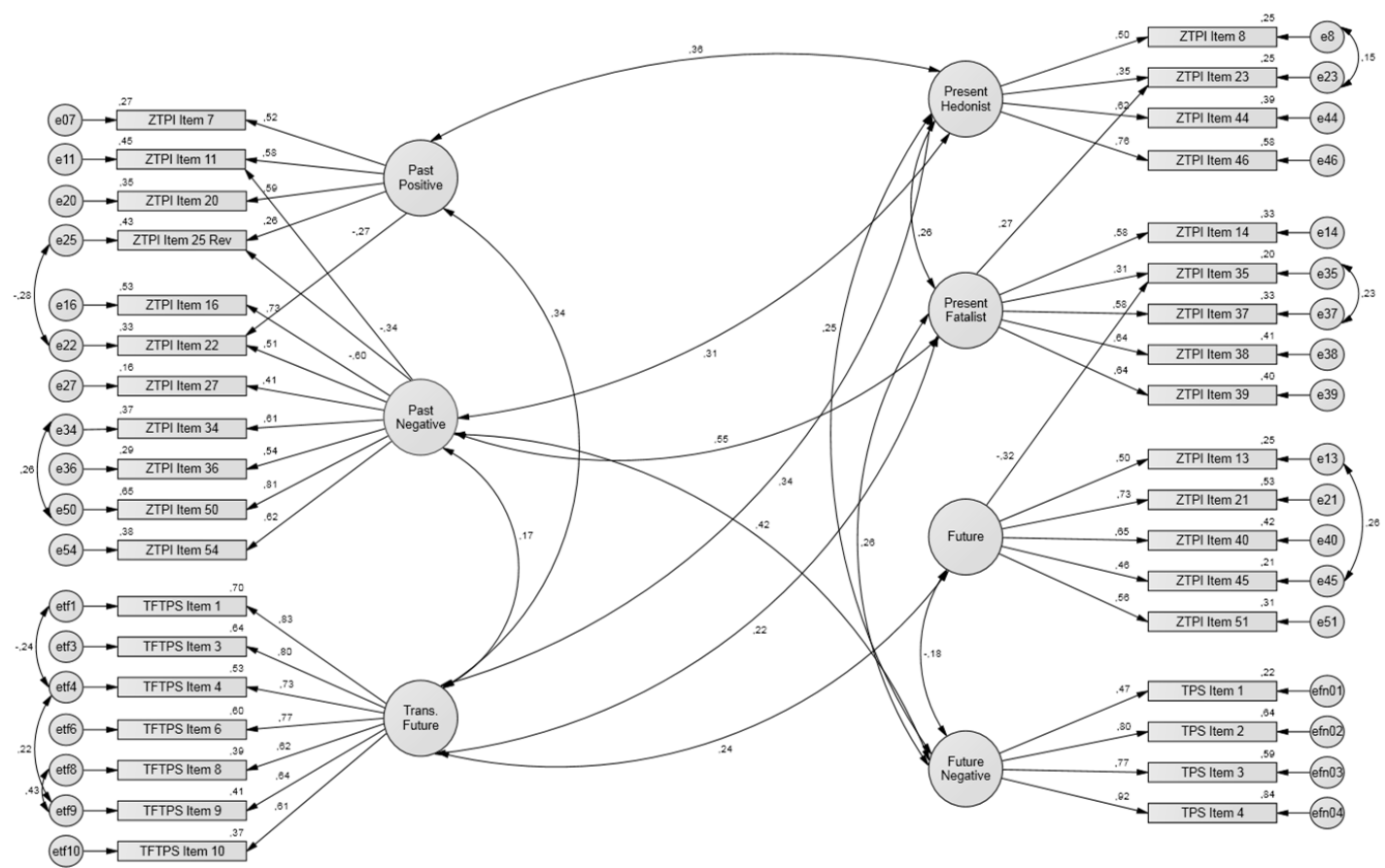

Figura 3. Path Diagram do modelo de PT com sete dimensões temporais

Nota. Índices de ajustamento global: $x^{2}(568)=895.30, p<.001 ; x^{2} / d f=1.58 ; \mathrm{CFI}=.88, \mathrm{PCFI}=.79 ; \mathrm{GF}=.82 ; \mathrm{PGFI}=.70$; $\mathrm{NFI}=.73 ; \mathrm{RMSEA}=.05, p=.31$.

estatisticamente ( $p>.05)$, nomeadamente: Passado Positivo <-> Passado Negativo, Presente Hedonista <-> Futuro, Presente Fatalista <-> Passado Positivo, Futuro <-> Passado Positivo, Futuro <-> Passado Negativo, Futuro <-> Presente Fatalista, Futuro Negativo <-> Passado Positivo e Futuro Transcendental <-> Futuro Negativo.

Os índices de ajustamento global do modelo estão muito próximos de um nível aceitável em alguns dos índices (CFI=.88), enquanto em outros (no caso dos índices de parcimónia) os resultados são aceitáveis (PCFI=.79). Os resultados mais detalhados acerca do ajustamento global do modelo, assim como o seu Path Diagram, podem ser consultado na Figura 3.

Como referido previamente para cada um dos inventários (Estudo 1 e Estudo 2), todos os itens incluídos no modelo final apresentaram uma associação estatisticamente significativa com o seu correspondente fator teórico. Também, as correlações entre os fatores são significativas ao nível $p<.05$. Todos os itens apresentam boa confiabilidade e cargas fatoriais. A única exceção é para a trajetória correlacional da TFTPS etf01 <$>$ etf03.

Posteriormente procedeu-se à Análise Fatorial Exploratória (AFE) com os itens escolhidos para o modelo final. $\mathrm{O}$ valor do índice Kaiser-
Meyer-Olkin (KMO) foi de .782, o que indica adequação da amostra para este tipo de análise. A variância total explicada foi de $56.20 \%$.

\section{Discussão}

O objetivo deste estudo foi testar através de uma técnica estatística robusta (Modelação de Equações Estruturais) a validade e confiabilidade de um modelo composto por sete dimensões temporais. Estas dimensões compreendem as cinco dimensões temporais do modelo apresentado por Zimbardo e Boyd (1999) e o Futuro Transcendental (Boyd \& Zimbardo, 1997). Estas seis dimensões, já foram apresentadas como um modelo conjunto por Zimbardo e Boyd (2008).

Contudo, tendo em conta a definição de PT proposta por Lewin (1965), assim como as considerações de outros autores em relação à influência que a temporalidade subjetiva futura possui no espaço individual psicológico e comportamental (Zaleski, 1996), foi decidido incluir uma dimensão relativa ao Futuro Negativo. Esta dimensão foi medida através a sub-dimensão Futuro Negativo/Visão Ansiosa de Futuro do Inventário de Perspetiva Temporal (Janeiro, 2012). 
O modelo teórico e estatístico de PT apresentado neste trabalho pode ser considerado como a evolução do modelo de 69 itens apresentado por Ortuño e colaboradores (2013c). O modelo atual utiliza menos itens e apresenta uma variância explicada total maior que o modelo apresentado por estes autores. Adicionalmente, o novo modelo de PT apresenta ainda elevados valores na carga fatorial do itens $(\lambda>.50)$, assim como alta confiabilidade $\left(R^{2}<.25\right)$.

Consideramos que a adição do Futuro Negativo ao modelo temporal, representa um importante contributo no estudo de psicopatologias, auto-estima (como já fora verificado por Ortuño, Paixão, \& Janeiro, 2013b, Ortuño \& Vásquez, 2013) e outras dimensões psicológicas importantes para a compreensão tanto do bem-estar como do mal-estar psicológico.

Globalmente, as alterações introduzidas no modelo resultaram numa maior consistência e confiabilidade do mesmo. Assim, nesta nova proposta, o modelo de PT é composto por 36 itens, os quais são agrupados em sete fatores, que representam 7 dimensões temporais únicas.

Grande parte das associações verificadas entre as sete dimensões temporais estão em conformidade com os resultados encontrados em estudos prévios. O Passado Positivo apresenta uma relação positiva com o Presente Hedonista (Zimbardo \& Boyd, 1999). Por outro lado, o Passado Negativo apresenta uma associação positiva tanto com o Presente Hedonista como com o Presente Fatalista (Zimbardo \& Boyd, 1999). O Presente Hedonista correlaciona-se positivamente com o Presente Fatalista (Zimbardo \& Boyd, 1999).

O Futuro Transcendental encontra-se correlacionado positivamente com o Passado Positivo, Passado Negativo, Presente Hedonista, Presente Fatalista e Futuro (Boyd \& Zimbardo, 1997). Já o Futuro Negativo, apresentou um padrão de correlações de acordo com os resultados prévios em alguns aspetos (relação positiva com Passado Negativo e Presente Fatalista), mas não noutros (relação negativa com Futuro e positiva com o Presente Hedonista), que foram reportados por Carelli e colaboradores (2011).

Todavia, existe uma relação que não foi explorada ainda por qualquer outro estudo. É o caso das dimensões Futuro Transcendental e Futuro Negativo. Conceitualmente, consideramos estas duas dimensões como diferentes, o Futuro Negativo é mais do que uma Orientação Temporal, já que possui também uma Valência Afetiva definida em relação ao futuro. Enquanto o Futuro Transcendental não possui qualquer valência afetiva em relação a esse marco temporal em específico (o futuro, após a morte do corpo físico). Podemos, pois, argumentar que o Futuro Transcendental não apresenta qualquer ligação afetiva ou emocional positiva ou negativa, já que não apresenta diferenças na sua associação com dimensões que revelam uma clara tendência positiva ou negativa. Caso disto, são o Passado Positivo e o Passado Negativo.

Consideramos que a ausência de associação entre o Futuro Transcendental e o Futuro Negativo pode dever-se, em parte ao conteúdo inerente a cada uma destas dimensões, já que por um lado o Futuro Transcendental se encontra relacionado com um possível futuro transcendente ou inclusive místico enquanto, por outro lado, o Futuro Negativo está relacionado com atividades diárias, objetivos, aspirações e medos em relação àquilo que Zimbardo e Boyd (2008) chamaram de "futuro mundano". Noutras palavras, não é mais de que uma expressão do que Casullo (1999) refere como a divisão na sociedade ocidental entre mundo do tempo e do espaço e o mundo do espiritual.

O modelo não apresentou uma relação expressiva ao nível estatístico entre estas duas dimensões ( $r_{\text {Futuro }}$ Transcendental-Futuro Negativo $=-.07$, $p=.329$ ), motivo pelo qual foi retirada do modelo. A decisão foi tomada tendo em conta apenas considerações estatísticas de forma a conseguir um modelo mais robusto, já que nas ideias apresentadas por Zimbardo e Boyd (1999) todas as dimensões temporais, ainda que independentes entre si, se encontram associadas.

Para além da sua validade estrutural e conceptual, o modelo de sete dimensões temporais apresenta uma quantidade considerável de variância explicada. Em estudos futuros será fundamental testar o poder preditivo deste mesmo modelo. Nesse aspeto, consideramos que, dada a natureza multidimensional do modelo proposto, este poderá apresentar importantes associações com uma ampla variedade de comportamentos e 
cognições, como já de facto ficou provado em alguns estudos que utilizaram um paradigma multidimensional (Carelli et al., 2011; Keough, Zimbardo, \& Boyd, 1999; Ortuño \& Vasquez, 2013).

\section{Conclusões Gerais}

A investigação na área da PT tem mostrado uma rápida evolução nos últimos anos. O número de publicações e eventos dedicados a esta particular dimensão da experiência subjetiva do tempo tem apresentado um incremento substancial.

Porém, o debate ainda se encontra em aberto em relação a importantes aspetos da PT, entre eles: i) a estrutura e composição da PT, já que diferentes autores se focam em diferentes dimensões desta, assim como também dão enfâse a determinados marcos temporais em detrimento de outros.

Em relação à AFC do ZTPI, a versão de 56 itens não apresentou bons indicadores. Contudo, uma versão reduzida de 25 itens apresentou bons valores na maioria dos índices de ajustamento global testados.

Em relação aos principais resultados obtidos na utilização da TFTPS, consideramos que este é um instrumento válido para utilização no contexto da investigação em Portugal. De facto, todos os indicadores estatísticos obtidos demonstram a qualidade desta escala, o que permite a sua utilização em diversos contextos - sejam normativos ou clínicos. Apesar disso, é necessário desenvolver mais investigação, de forma a estabelecer qual a utilidade preditiva desta escala.

Tendo em conta o conteúdo presente nesta escala, acreditamos que grande parte do seu valor preditivo poderá ser encontrado em populações idosas ou junto de grupos com fortes crenças religiosas e/ou espirituais, isto, dado a maior proximidade do fim da vida física (nos idosos) e a forte relação que as crenças religiosas apresentam com os eventos que são antecipados acerca da vida mais-além da morte física.

O novo modelo com sete dimensões temporais apresentou resultados muito positivos tanto a nível exploratório como confirmatório. No caso específico da AFC, é importante considerar que os índices de ajustamento globais que não são afetados por variáveis como o número da amostra ou o número de parâmetros do modelo, são aqueles onde foram obtidos os melhores resultados. Caso disto, são os índices de parcimónia (PCFI e PGFI) ou o chi quadrado dividido pelo número de graus de liberdade $\left(X^{2} / d f\right)$, abordagem que já fora mencionada por Zimbardo e Boyd (1999), tendo em conta considerações metodológicas previamente propostas por outros autores.

Contudo, não podemos deixar de mencionar a maior limitação deste estudo, a qual é a composição de participantes, os quais são na sua generalidade estudantes universitários, o que limita a possibilidade de generalização destes resultados e, em última análise, do modelo aqui apresentado. Recomendamos que futuros estudos incluam amostras mais heterogéneas a fim de colmatar esta limitação. Outro aspecto importante a considerar é ao nível da estrutura da temporalidade subjetiva, já que neste estudo apenas é abordada a Perspectiva Temporal (ou Orientação Temporal nos termos apresentados por Nuttin \& Lens, 1985). Devem ser incluídos em futuros modelos outros instrumentos que avaliem diferentes dimensões da temporalidade subjetiva igualmente importantes na compreensão do comportamento humano (por exemplo a Extensão Temporal).

Em relação à nossa proposta de um novo modelo multidimensional da PT, consideramos ser um passo em frente em relação à estrutura conceptual e fatorial da PT, ao considerar uma importante dimensão da temporalidade subjetiva como é o Futuro Negativo. A importância desta dimensão já tinha sido demostrada previamente noutros estudos (Carelli et al., 2011, Ortuño \& Vásquez, 2013, Zaleski, 1996), com diversos métodos de avaliação, o que fortalece esta mesma evidência.

\section{Referências}

Anagnostopoulos, F., \& Griva, F. (2012). Exploring time perspective in greek young adults: Validation of the Zimbardo Time Perspective Inventory and relationships with mental health indicators. Social Indicators 
Research, 106(1), 41-59. doi:10.1007/s11205011-9792-y

Apostolidis, T., \& Fieulaine, N. (2004). Validation française de l'échelle de temporalité The Zimbardo Time Perspective Inventory (ZTPI). Revue européenne de psychologie appliquée, 54(3), 207-217. doi: 10.1080/17439760903271181

Azevedo, M. J., Teixeira, L., \& Paúl, C. (2012). Development and validation of a brief ZTPI for Portuguese older people. Poster apresentado na Ist International Conference on Time Perspective. Coimbra: Portugal.

Boyd, J. N., \& Zimbardo, P. G. (1997). Constructing time after death: The transcendental future time perspective. Time and Society, 6(1), 35-54. doi:10.1177/0961463X97006001002

Carelli, M. G., Wiberg, B., \& Wiberg, M. (2011). Development and construct validation of the Swedish Zimbardo Time Perspective Inventory. European Journal of Psychological Assessment, 27(4), 220-227. doi:10.1027/101 5-5759/a000076

Carvalho, R. G. G. (2015). Future time perspective as a predictor of adolescents' adaptive behavior in school. School Psychology International, 36(5), 482-497. doi:10.1177/0143034315601167

Casullo, M. M. (1999). La evaluación psicológica: Modelos, técnicas y contexto sociocultural. Revista Iberoamericana de Diagnóstico y Evaluación - e Avaliação Psicológica, 1, 97-113.

Corral-Verdugo, V., Fraijo-Sing, B., \& Pinheiro, J. (2006). Sustainable behavior and time perspective: Present, past and future orientations and their relationship with water conservation behavior. Interamerican Journal of Psychology, 40, 139-147. doi: $10.1177 / 0013916512474987$

Cretu, R. Z. (2012). A Confirmatory Approach of the Structure of Zimbardo's Time Perspective Concept. Cognition, Brain, Behavior, XVI(4), 481-494.

Diaz-Morales, J. F. (2006). Estructura factorial y fiabilidad del Inventario de Perspectiva Temporal de Zimbardo. Psicothema, 18(3), 565-571.
Ferrari, J. R., \& Díaz-Morales, J. F. (2007). Procrastination: Different time orientations reflect different motives. Journal of Research in Personality, 41(3), 707-714. doi:10.1016/j.jrp.2006.06.006

Gupta, R., Hershey, D. A., \& Gaur, J. (2012). Time Perspective and Procrastination in the Workplace: An Empirical Investigation. Current Psychology, 31, 195-211. doi:10.1007/s12144-012-9136-3

Holman, E. A., \& Silver, R. C. (2005). Futureoriented thinking and adjustment in a nationwide longitudinal study following the September 11th Terrorist Attacks. Motivation and Emotion, 29(4), 389-410. doi:10.1007/s11031-006-9018-9

Hooper, D., Coughlan, J., \& Mullen, M. R. (2008). Structural equation modelling: Guidelines for determining model fit. The Electronic Journal of Business Research Methods, 6(1), 53-60.

Imaginário, S., Oyanadel, C., Gamboa, V., \& Jesus, S. N. (2011). Qualidades psicométricas da adaptação para português do Zimbardo Time Perspective Inventory - Versão Reduzida (ZTPI-R). In A. Ferreira, A. Verhaeghe, D. Silva, L. Almeida, R. Lima, \& S. Fraga (Eds.), Actas del VIII Congresso Iberoamericano de Avaliação Psicológica (pp. 1735-1746). Lisboa: Universidade de Lisboa.

Janeiro, I. N. (2012). O Inventário de Perspectiva Temporal: Estudo de validação. Revista Iberoamericana de Diagnóstico e Avaliação e Avaliação Psicológica, 34, 117-133.

Keough, K. A., Zimbardo, P. G., \& Boyd, J. N. (1999). Who's Smoking, Drinking, and Using Drugs? Time Perspective as a Predictor of Substance Use. Basic and Applied Psychology, 21, 149-164. doi:10.1207/S1532 4834BA210207

Koštál, J., Klicperová-Baker, M., Lukavská, K., \& Lukavský, J. (2015). Short version of the Zimbardo Time Perspective Inventory (ZTPIshort) with and without the Future-Negative scale, verified on nationally representative samples. Time \& Society. doi:10.1177/09614 63X15577254

Leite, U. R., \& Pasquali, L. (2008). Estudo de Validação do Inventário de Perspectiva de 
Tempo do Zimbardo. Avaliação Psicológica, 7(3), 301-320.

Lewin, K. (1939). Field theory and experiment in social psychology: Concepts and methods. American journal of sociology, 44(6), 868896.

Lewin, K. (1965). Teoria de Campo em Ciência Social. (C. M. Bori, Trad.). São Paulo: Livraria Pioneira Editora. (Trabalho original publicado em 1951).

Liniauskaite, A., \& Kairys, A. (2009). The Lithuanian version of the Zimbardo Time Perspective Inventory (ZTPI). Psichologija, 40, 66-87.

Marôco, J. (2010). Análise de equações estruturais: Fundamentos teóricos, Software \& Aplicações. Pêro Pinheiro: ReportNumber.

Milfont, T. L., \& Gouveia, V. V. (2006). Time Perspective: An exploratory study of their relations to environmental attitudes. Journal of Environmental Psychology, 26, 72-82. doi:10.1016/j.jenvp.2006.03.001

Milfont, T. L., Andrade, T. L., Belo, R. P., \& Pessoa, V. S. (2008). Testing Zimbardo time perspective inventory in a Brazilian sample. Interamerican Journal of Psychology, 42, 4958.

Nobre, A. S., \& Janeiro, I. N. (2010). Relação entre a Perspectiva Temporal e a Adaptação à Escola em alunos do $9^{\circ}$ ano. In C. Nogueira, I. Silva, L. Lima, A. T. Almeida, R. Cabecinhas, R. Gomes, C. Machado, A. Maia, A. Sampaio, \& M. C. Taveira (Eds.), Actas do VII Simpósio Nacional de Investigação em Psicologia (pp. 3033-3043). Braga: Universidade do Minho.

Nuttin, J., \& Lens, W. (1985). Future Time Perspective and Motivation: Theory and research method. Belgium: Leuven University Press.

Orkibi, H. (2015). Psychometric properties of the Hebrew short version of the Zimbardo Time Perspective Inventory. Evaluation \& The Health Professions, 38(2), 219-245. doi:10.1177/0163278714531601

Ortuño, V. (2014). Time perspective stability: Studies with a multidimensional model in the university context. Tese de Doutoramento não publicada. Universidade de Coimbra, Coimbra, Portugal.
Ortuño, V., \& Gamboa, V. (2008). Estudo Preliminar de Adaptação ao Português do Zimbardo Time Perspective Inventory - ZTPI. In A. Noronha, C. Machado, L. Almeida, M. Gonçalves, S. Martins, \& V. Ramalho (Eds.), Actas da XIII Conferência Internacional de Avaliação Psicológica: Formas e Contextos. Braga: Universidade do Minho.

Ortuño, V. E., \& Gamboa, V. M. (2009). Estrutura factorial do Zimbardo Time Perspective Inventory - ZTPI numa amostra de estudantes universitários portugueses. Avances en Psicología Latinoamericana, 27(1), 21-32.

Ortuño, V., \& Vásquez Echeverría, A. (2013). Time Perspective and Self-Esteem: Negative Temporality Affects the Way We Judge Ourselves. Annales Universitatis Paedagogicae Cracoviensis. Studia Psychologica, VI, 109-125.

Ortuño, V. E., Paixão, M. P., \& Janeiro, I. N. (2013a). Tempus Post Mortem? Adaptação Portuguesa da Transcendental-Future Time Perspective Scale - TFTPS. Avances en Psicologia Latinoamericana, 31(2), 396-408.

Ortuño, V., Paixão, M. P., \& Janeiro, I. (2013b) O tempo subjectivo como instrumento instrumento (des)adaptativo no processo desenvolvimental. Análise Psicológica, 2(XXXI), 159-169.

Ortuño, V., Gomes, C., Paixão, M. P., \& Janeiro, I. (2013c). An exploratory approach to Time Perspective theory and research. In M. P. Paixão, J. T. da Silva, V. Ortuño, \& P. Cordeiro (Eds.), International Studies on Time Perspective (pp. 247-252). Coimbra: University of Coimbra Press. doi:10.13140/RG.2.1.1602.0008

Ortuño, V., Gomes, C., Vásquez, A., Belo, P., Imaginário, S., Paixão, M. P., \& Janeiro, I. (2013d). Satisfaction with life and college social integration: A Time Perspective multiple regression model. In M. P. Paixão, J. T. da Silva, V. Ortuño, \& P. Cordeiro (Eds.), International Studies on Time Perspective (pp. 101-106). Coimbra: University of Coimbra Press. doi:10.13140/RG.2.1.1602.0008

Sircova, A., van de Vijver, F., Osin, E., Milfont, T., Fieulaine, N., Kislali-Erginbilgic, A., Zimbardo, P. \& 54 members of the 
International Time Perspective Research Project (2014). A global look at time: A 24 country study of the equivalence of the Zimbardo Time Perspective Inventory. SAGE Open, 4(1), 1-12. doi:10.1177/215824401351 5686

Trommsdorff, G. (1983). Future orientation and socialization. International Journal of Psychology, 18, 381-406. doi:10.1080/002075 98308247489

Zaleski, Z. (1996). Future Anxiety: Concept, measurement, and preliminary research. Personality and Individual Differences, 21(2), 165-174.

Zimbardo, P. G., \& Boyd, J. N. (1999). Putting time in perspective: A valid, reliable individual differences metric. Journal of Personality and Social Psychology, 77, 12711288. doi:10.1037/0022-3514.77.6.1271

Zimbardo, P. G., \& Boyd, J. N. (2008). The time paradox: Using the New Psychology of Time to Your Advantage. London: Rider. 